\title{
Narrow band imaging efficiency in evaluation of mucosal healing/ relapse of ulcerative colitis
}

\section{다우우}

Authors

Seiko Sasanuma ${ }^{1}$, Kazuo Ohtsuka ${ }^{1}, 2$, Shin-ei Kudo ${ }^{1}$, Noriyuki Ogata ${ }^{1}$, Yasuharu Maeda ${ }^{1}$, Masashi Misawa ${ }^{1}$, Yuichi Mori $^{1}$, Toyoki Kudo' ${ }^{1}$, Tomokauzu Hisayuki ${ }^{1}$, Kunihiko Wakamura ${ }^{1}$, Takemasa Hayashi' ${ }^{1}$, Atsushi Katagiri ${ }^{1}$, Hideyuki Miyachi $^{1}$, Toshiyuki Baba ${ }^{1}$, Fumio Ishida ${ }^{1}$

Institutions

1 Digestive Disease Center, Showa University Northern Yokohama Hospital, Yokohama, Japan

2 Department of Endoscopy, Tokyo Medical and Dental University, Tokyo, Japan

submitted 18.9.2017

accepted after revision 10.1.2018

Bibliography

DOI https://doi.org/10.1055/s-0044-102297 |

Endoscopy International Open 2018; 06: E518-E523

(c) Georg Thieme Verlag KG Stuttgart · New York ISSN 2364-3722

Corresponding author

Seiko Sasanuma (Hayashi), Showa University Northern

Yokohama Hospital - Digestive Disease Center, 35-1

Chigasaki-chuo Tsuzuki, Yokohama Kanagawa 224-8503,

Japan

Fax: +81-45-949-7263

sei_hy_8@yahoo.co.jp

\section{ABSTRACT}

Background and study aims Mucosal healing is a current treatment target in ulcerative colitis (UC), while histological remission is another target. The aim of this study was to evaluate the efficiency of magnified narrow band imaging (NBI) findings of mucosal healing and their relationship with histological activity and prognosis.

Patients and methods Patients with UC who underwent total colonoscopy between January 2010 and December 2012 with left-sided or total-colitis type UC and achieved clinical remission with an endoscopic Mayo score of 0 or 1 were included. Each colon section was observed with white light and magnified NBI, with the colonoscopy being repeated at 1-year follow-up. We assessed the relationships of magnified NBI with histological disease activity and prognosis. Magnified NBI findings were divided into three categories; honeycomb-like blood vessels (BV-H), blood vessels shaped like bare branches (BV-BB), and blood vessels shaped like vines (BV-V).

Results Fifty-two patients were included. The percentage of remitted mucosa with BV-BB was $37 \%$, while that of mucosa with scars with BV-H was $35 \%$. BV-H and BV-BB did not show pathological activity (12/292 and 8/299, respectively), while BV-V showed high pathological activity $(27 / 33$, $81 \%)$. There was a correlation between magnified NBI findings and pathological findings $(P<0.01)$. The odds ratio for inflammation activity at 1-year follow-up was 14.2 for BVBB $(95 \% \mathrm{Cl}, 3.3-60.9)$

Conclusion Magnified NBI findings showed a good relationship with histological activity. This suggests that we could estimate histological activity without biopsy, and also the possibility of predicting relapse over the following year.

\section{Introduction}

Advances in treatment of ulcerative colitis (UC) have changed the treatment target $[1,2]$. Consensus guidelines for clinical practice and trial endpoints recommend achieving endoscopic mucosal healing, not just clinical remission [1,3]. Endoscopic mucosal healing is defined as resolution of visible mucosal inflammation and ulceration. Achievement of endoscopic mucosal healing is associated with clinical remission and lower rates of hospitalization and operations [4-6]. Several reports have shown that mucosa that had seemingly reached mucosal healing still had a slight histologically-defined inflammation and a possibility for relapse [7-9]. Therefore, an approach using assessment of mucosal healing is necessary [10]. Following endoscopic mucosal healing, histological remission becomes the next treatment target; a recent report showed that histological remission was a better predictor of corticosteroid use and hospitalization [11].

Several scoring systems for evaluation of severity of inflammation have been reported, with endoscopic assessment play- 
ing an important role. The Mayo scoring system is commonly used, and Mayo scores of both 0 and 1 are defined as mucosal healing [12]. High-resolution magnifying colonoscopy has enabled detailed observation of the surface of colorectal mucosa. Narrow band imaging (NBI) is one such modality, with the NBI findings of UC mucosa having been reported in previous studies that evaluated both vascular patterns and mucosal membrane structure together [13-15].

In this study, we utilized the characteristics of NBI to observe the capillary vessels, and focused on the vascular patterns of the mucosa on magnified NBI. We assessed them not only in the rectum, but in all segments of the colon. The aim of this study was to analyze the value of magnified NBI findings of mucosal healing, evaluate them in relation to histological activity, and detect differences in prognosis, for improvements in clinical use.

\section{Patient and methods}

\section{Patients}

Colonoscopies were performed on patients with UC from January 2010 to December 2012 at our institute. Patients were included in this study if they had undergone a total colonoscopy with left-sided or total-colitis type UC, were in clinical remission, and had an endoscopic Mayo score of 0 or 1 . Lack of clinical remission, rectal type UC, and a past history of colectomy were exclusion criteria.

\section{Instrument}

Magnifying colonoscopy (CF-H260AZI, Olympus, Tokyo, Japan) was used for the examinations. The endoscope unit used was an EVIS LUCERA ELITE system (Olympus). When insertion was difficult because of pain or stiffness, a PCF-H260ZI scope was used with $\mathrm{CO}_{2}$ insufflation.

\section{Procedure}

Total colonoscopy was performed with white light and magnifying NBI to observe the mucosa of each segment of the colorectum. For the purpose of assessment, the colorectum was divided into six segments, defined as the cecum, ascending colon, transverse colon, descending colon, sigmoid colon, and rectum. A biopsy specimen was taken from each segment of the mucosa. After 1 year, a repeat colonoscopy was performed in the same manner as the original procedure.

We first defined the magnified NBI findings as three categories, as described below. The findings on white light and NBI were compared, with the white light images being divided into two groups: remitted mucosa with or without scars. We first assessed this to know if the standard classification of white light can predict NBI findings, which seemed to be just as mucosal healing and no other findings at a glance. Then, the pathological results from all of the biopsy specimens were compared with the results of the magnified NBI findings to assess the relationship between imaging and pathological activity. The relationships between the initial and 1-year follow-up NBI findings were assessed to reveal the different prognoses for the NBI findings.
This study was approved by the hospital ethics committee (Showa University Northern Yokohama Hospital, 16H006) and informed consent was obtained before examination.

\section{NBI findings}

Those cases with a Mayo score of 0 and 1 showed some specific findings on magnifying NBI images. These NBI findings were newly divided into three types, according to their blood vessels. These types were honeycomb-like blood vessels (BV-H), where healthy hexagon-shaped vessels were visible; blood vessels shaped like bare branches (BV-BB), where only thin vessels were visible, and blood vessels shaped like vines (BV-V), where slightly thicker winding vessels were visible ( $\triangleright$ Fig. 1 ). When the characteristics of more than one type were present in the same area, the higher grade was recorded.

\section{Histological assessment}

Mucosal biopsies were taken from each part of the colon. Biopsy specimens were fixed in $10 \%$ neutral-buffered formalin, embedded in paraffin, and sectioned into $4-\mu \mathrm{m}$ slices. Hematoxylin and eosin staining was used for histopathological examination. The pathologists, who belonged to the hospital pathology department, were blinded to the endoscopic findings. Definitions for histological findings followed those of the Japanese Ministry of Health, Labour, and Welfare. Existence of active inflammation was determined according to existence of one of three items: (1) diffuse infiltration of inflammatory cells; (2) erosion; and (3) a crypt abscess/cryptitis.

\section{Statistical methods}

Statistical analysis of the results of the pathological findings was performed using Fisher's exact test. The upper and lower $95 \%$ confidence intervals $(\mathrm{Cl})$ were calculated based on a binominal distribution. A $P$ value $<0.05$ was considered statistically significant. All statistical analyses were performed using R software version 3.2.2 (R Foundation for Statistical Computing, Vienna, Austria).

\section{Results}

A total of 185 UC patients underwent initial colonoscopy, with 52 patients fulfilling the inclusion criteria who underwent a second 1-year-follow-up colonoscopy and were enrolled in this study ( $\triangleright$ Fig. 2 ). Patient characteristics are shown in $\triangleright$ Table 1. In this study, most patients were treated with 5ASA at the remission phase.

Biopsy specimens were taken from each segment of the mucosa, giving a total of 312 samples.

\section{Inconsistency in findings of white light and NBI}

First, we compared the findings from white light and NBI images ( Fig.3). More than $60 \%$ of mucosa with a normal appearance on white light showed BV-H on NBI. Two-thirds of mucosa with scars on white light showed BV-BB on NBI ( $>$ Table 2). 


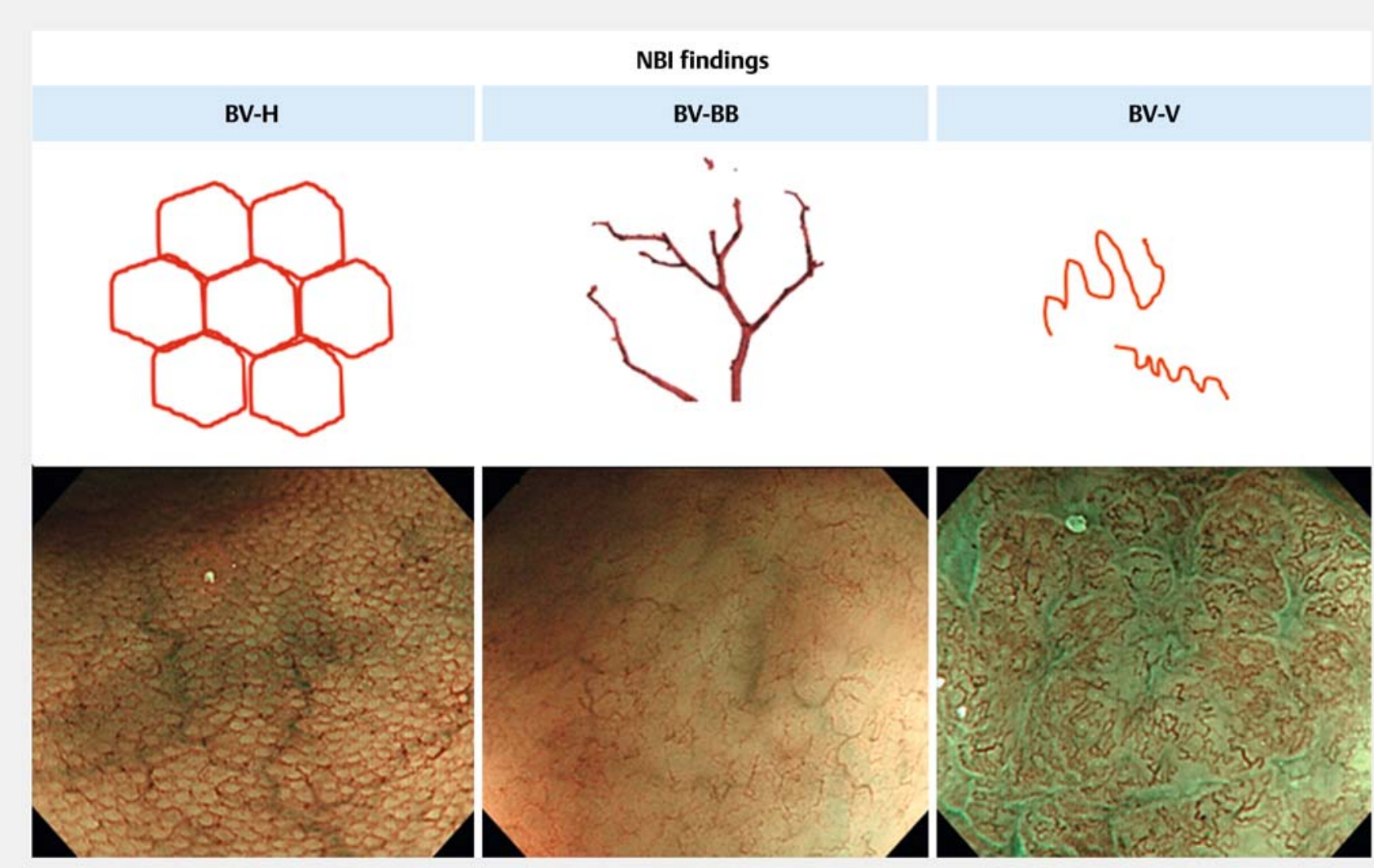

Fig. 1 Classification of the NBI findings. BV-H, blood vessels with honeycomb-like appearance; BV-BB, blood vessels shaped like bare branches; BV-V, blood vessels shaped like vines.

\section{Patients}

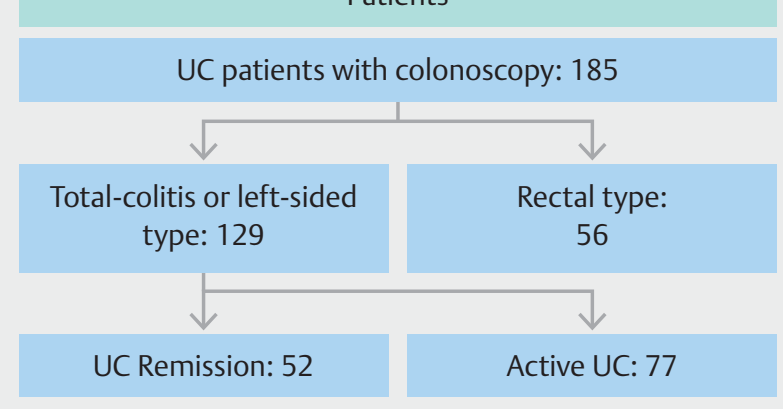

Fig. 2 Study flow chart.

\section{NBI findings and histology}

Results of histological findings from the examined lesions are shown in Table 3. NBI BV-H (12/292 showing activity: 4\%) and NBI BV-BB (8/299 showing activity: $3 \%)$ rarely showed activity on histology. To the contrary, a classification of NBI BV-V generally indicated pathological activity (27/33, $81 \%)$. There was a significant correlation between magnified NBI findings and histological findings $(P<0.001)$.

\begin{tabular}{|l|l|l|}
\hline \multicolumn{1}{|l|}{ Table 1 Patients characteristics } & & \\
\hline Characteristic & $\begin{array}{l}\text { Total colitis } \\
\text { type }\end{array}$ & $\begin{array}{c}\text { Left sided } \\
\text { type }\end{array}$ \\
\hline Number of patients & 33 & 19 \\
\hline Gender Male/Female & $15 / 18$ & $11 / 18$ \\
\hline Mean age \pm SD & $50.3 \pm 15.6$ & $49.2 \pm 14.8$ \\
\hline Mean duration of disease(years) \pm SD & $9.7 \pm 7.8$ & $9.1 \pm 7.4$ \\
\hline Mayo 0/1 & $25 / 8$ & $16 / 3$ \\
\hline Treatment & & 14 \\
\hline - 5-ASA mesalazine & 30 & 3 \\
\hline - Salazosulfapyridine & 2 & 0 \\
\hline - Prednisolone & 1 & 1 \\
\hline - Immunomodulator & 0 & 0 \\
\hline - Biologics & 1 & 2 \\
\hline - No medication & & 0 \\
\hline
\end{tabular}




\section{White light imges and NBI}
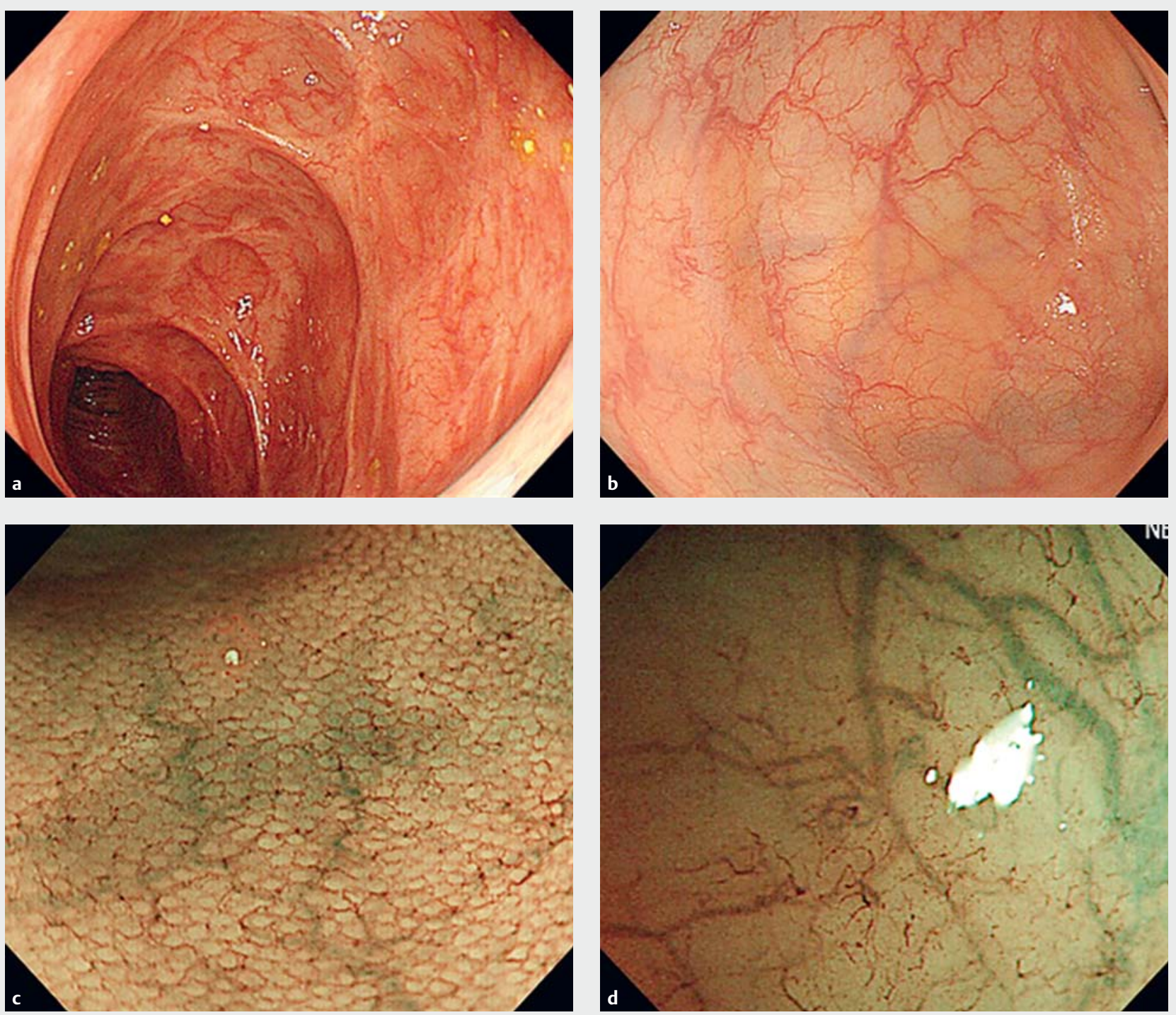

- Fig. 3 White light images and magnified NBI images. a White light image showing remitted mucosa with scarring. b White light image showing remitted mucosa without scarring. $\mathbf{c}$ Magnified NBI of mucosa in a. $\mathbf{d}$ Magnified NBI of mucosa in $\mathbf{b}$.

Interval changes in the NBI findings

NBI findings for the first examination revealed 144 lesions with $\mathrm{BV}-\mathrm{H}, 162$ with BV-BB, and 6 with BV-V. The NBI findings for the second 1-year follow-up examination showed 148 lesions with BV-H, 137 with BV-BB, and 27 with BV-V ( $\bullet$ Table 4).

Of 144 lesions with NBI BV-H, two were histologically active the following year, while among 162 lesions with NBI BV-BB, 27 lesions were histologically active at 1-year follow-up. The odds ratio for NBI BV-BB showing active inflammation at 1-year follow-up was $14.2(95 \% \mathrm{Cl}, 3.3-60.9)$ ( $\triangleright$ Table 5). On the other hand, three BV-V lesions were spontaneously improved the 1-year follow-up.

Clinical recurrence was observed in 10 cases, with treatment being intensified for these patients.

\section{Discussion}

This study showed effective results for investigations using NBI findings in the remission phase, and for predicting risk of recurrence of UC. First, we compared the white light images and magnified NBI images of the remission phase (Mayo 0 or 1 ) and found the results showed a discrepancy. Second, we focused on the shapes of capillary vessels with magnifying NBI and showed a good correlation between findings from NBI findings and histology. Third, the results revealed that cases showing NBI BV-BB had a higher risk of recurrence than those showing $\mathrm{NBI} \mathrm{BV}-\mathrm{H}$; this indicated that the difference in NBI findings reflected a different prognosis. 
In this study, the comparison between the white light images and magnified NBI images of UC remission showed a discrepancy. Vascular pattern, mucosal friability, and bleeding were included as evaluation items for the major previous endoscopic grading of UC, as they were recognized as important factors [8, 16]. Recently, another endoscopic score was proposed: the UC Endoscopic Index of Severity (UCEIS), which also included the above-mentioned factors [17]. The vascular pattern of "normal" on the white light images was defined as a normal vascular pattern with arborization of capillaries clearly defined, or with blurring or patchy loss of capillary margins [17]. Our study results showed that some of the UC mucosa that had seemingly reached mucosal healing had different vascular patterns with magnification. This demonstrates the limits of the scoring system in evaluation of mucosal healing and subtle residual inflammation. Vascular patterns have been recognized as an important factor, and we think it is better to assess these patterns more carefully with magnification, when they are visible.

The results of our study showed that there was a correlation between the magnifying NBI findings and histological findings. As for the vasculature, mucosal angiogenesis plays an important role in neoplastic and non-neoplastic chronic inflammatory disorders $[13,18,19]$. Previous reports have documented an observed increase in vascular density in IBD, which directly correlated with increased IBD disease severity [18]. In our results, histologically inactive areas showed only thin vessels or sparsely visible vessels. Recently, endocytoscopy (Olympus, Tokyo) has been developed as a virtual histology method [20]. This enables real-time observation of cells and nuclei in vivo using $450 \times$ ultra-magnification. Maeda et al assessed the capillaries in rectal mucosa with endocytoscopic NBI (EC-NBI) [21] and found that EC-NBI findings were correlated with histological inflammation and aided in differential diagnosis between active and inactive UC without biopsy. Unfortunately, this technique is still not widely available.

A notable finding in this study was that the different NBI vascular patterns had different risks of recurrence. Previously, Nishio et al reported on assessment of rectal mucosa with magnifying colonoscopy and reported that the grading scale had a good correlation with histological findings [22]. They also assessed prediction of the relapse of UC. To the contrary, rectal mucosa do not always reflect inflammation under the treatment intervention. Therefore, we assessed the whole length of the colonic mucosa in this study. Another high-definition colonoscopy system, the i-Scan (Pentax medical, Tokyo), also has the ability to enhance images $[23,24]$. lacucci et al observed whole segments of the colon and reported a good relationship between the i-Scan grading scale and histological activity [23]. Unfortunately, the report did not reveal information on prognosis. The findings for NBI-BB suggest a need for careful observation and treatment.

This study has several limitations, including the single center and small sample size, especially for NBI VB-B. Because patients in this study were mainly treated with 5-ASA alone, selection bias occurred. Long-term prognosis over more than 1 year was not analyzed. A further limitation is that the clinical endpoint
- Table 2 Results from white light images and magnified NBI findings.

\begin{tabular}{|l|r|l|l|}
\hline White light images & \multicolumn{3}{|c|}{ Magnified NBI findings } \\
\hline & \multicolumn{1}{|l|}{ BV-H } & \multicolumn{1}{|l|}{ BV-BB } & Total lesions \\
\hline Remitted mucosa & $100(63.3 \%)$ & $58(36.7 \%)$ & 158 \\
\hline Mucosa with scar & $52(35.1 \%)$ & $96(64.9 \%)$ & 148 \\
\hline
\end{tabular}

Table 3 Results of whole pathological findings.

\begin{tabular}{|l|c|c|c|}
\hline Pathological findings & \multicolumn{2}{|c|}{ NBI findings } \\
\hline & BV-H & BV-BB & BV-V \\
\hline Active inflammation (+) & $12(4 \%)$ & $9(3 \%)$ & $25(81 \%)$ \\
\hline Active inflammation (-) & $280(96 \%)$ & $291(97 \%)$ & $6(19 \%)$ \\
\hline
\end{tabular}

- Table 4 NBI findings for the first year and after 1 -year.

\begin{tabular}{|l|l|l|c|}
\hline \multicolumn{3}{|l|}{ NBI findings } \\
\hline & BV-H & BV-BB & BV-V \\
\hline First year & $144(46 \%)$ & $162(52 \%)$ & $6(2 \%)$ \\
\hline 1-year follow-up & $148(47 \%)$ & $137(44 \%)$ & $27(8.7 \%)$ \\
\hline
\end{tabular}

- Table 5 Interval change of NBI, BV-BB, and BV-H to BV-V after 1-year follow-up.

\begin{tabular}{|c|c|c|}
\hline & Histologically active & Histologically inactive \\
\hline BV-BB & 27 & 135 \\
\hline BV-H & 2 & 142 \\
\hline
\end{tabular}

was not compared with a definitive indicator such as total colectomy.

\section{Conclusion}

In conclusion, our analysis suggests that magnifying NBI observation is effective for assessment of UC follow-up. In cases with blood vessels shaped like bare branches, careful observation for relapse of UC is necessary.

\section{Acknowledgements}

We thank Karl Embleton, PhD, from Edanz Group for English editing a draft of this manuscript.

\section{Competing interests}

\section{None}




\section{References}

[1] Travis SP, Haggins PD, Orchard T et al. Review article: defining remission in ulcerative colitis. Aliment Pharmacol Ther 2011; 34: 113-124

[2] Peyrin-Biroulet L, Sandborn W, Sands BE et al. Selecting therapeutic targets in inflammatory bowel disease (STRIDE). Am J Gastroenterol 2015; $110: 1324-38$

[3] Peyrin-Biroulet L, Ferrante M, Magro F et al. Results from the $2^{\text {nd }}$ Scientific Workshop of the ECCO (I): Impact of mucosal healing on the course of inflammatory bowel disease. J Crohns Colitis 2011; 5: 477483

[4] Rutgeerts P, Vermeire S, Van Assche G et al. Mucosal healing in inflammatory bowel disease: impossible ideal or therapeutic target? Gut 2007; 56: $453-455$

[5] Sandborn W], Hanauer S, Van Assche G et al. Treating beyond symptoms with a review to improving patient outcomes in inflammatory bowel diseases. J Crohns Colitis 2014; 8: 927 - 935

[6] Colombel JF, Rutgeerts P, Reinsch W et al. Early mucosal healing with infliximab is associated with improved long-term clinical outcomes in ulcerative colitis. Gastroenterology 2011; 141: 1194-1201

[7] Danese S, Fiorino G, Angelucci E et al. Narrow-band imaging endoscopy to assess mucosal angiogenesis in inflammatory bowel disease: A pilot study. World J Gastroenterol 2010; 16: $2396-2400$

[8] Pousa ID, Gisbert JM, Gisbert JP et al. Angiogenesis in inflammatory bowel disease. Eur ] Clin Invest 2008; 38: 73-81

[9] Adachi S, Arakawa H, Koyama S et al. Correlation between endoscopic healing and histological healing of ulcerative colitis determined using the Mayo score. Progress Digest Endosc 2014; 85: 43-46

[10] Samaan MA, Mosli MH, Sandborn WL et al. A systematic review of the measurement of endoscopic healing in ulcerative colitis clinical trials: Recommendations and implications for future research. Inflamm Bowel Dis 2014; 20: $1465-1771$

[11] Bryant RV, Burger DC, Delo J et al. Beyond endoscopic mucosal healing in UC: histological remission better predicts corticosteroid use and hospitalization over 6 years of follow-up. Gut 2016; 65: 408-414

[12] D’Haens G, Sandborn W], Feagan BG et al. A review of activity indices and efficacy end points for clinical trials of medical therapy in adults with ulcerative colitis. Gastroenterology 2007; 132: $763-786$
[13] Kudo T, Matsumoto T, Esaki M et al. Mucosal vascular pattern in ulcerative colitis: observations using narrow band imaging colonoscopy with special reference to histologic inflammation. Int J Colorectal Dis 2009; 24: 495- 501

[14] Esaki M, Kubokura N, Kudo T et al. Endoscopic findings under narrow band imaging colonoscopy in ulcerative colitis. Digest Endosc 2011; 23: $140-142$

[15] Matsumoto T, Kudo T, Jo Y et al. Magnifying colonoscopy with narrow band imaging system for the diagnosis of dysplasia in ulcerative colitis: A pilot study. Gastrointest Endosc 2007; 66: 957 - 965

[16] lacucci M, Ghosh S. Looking beyond symptom relief: evaluation of mucosal healing in inflammatory bowel disease. Therap Adv Gastroenterol 2011; 4: 129-143

[17] Travis SP, Schnell D, Krzeski P et al. Developing an instrument to assess the endoscopic severity of ulcerative colitis: the Ulcerative Colitis Endoscopic Index of Severity (UCEIS). Gut 2012; 61: 535-642

[18] Cromer WE, Mathis JM, Granger DN et al. Role of the endothelium in inflammatory bowel diseases. World J Gastroenterol 2011; 17: 578 593

[19] Danese S, Sans M, Motte C et al. Angiogenesis as a novel component of inflammatory bowel disease pathogenesis. Gastroenterology 2006; 130: 2060 - 2073

[20] Bessho R, Kanai T, Hosoe $\mathrm{N}$ et al. Correlation between endocytoscopy and conventional histopathology in microstructural features of ulcerative colitis. J Gastroenterol 2011; 46: 1197-1202

[21] Maeda Y, Ohtsuka K, Kudo SE et al. Endocytoscopic narrow-band imaging efficiency for evaluation of inflammatory activity in ulcerative colitis. World ] Gastroenterol 2015; 21: 2108-2115

[22] Nishio $\mathrm{Y}$, Ando T, Maeda $\mathrm{O}$ et al. Pit patterns in rectal mucosa assessed by magnifying colonoscope are predictive of relapse in patients with quiescent ulcerative colitis. Gut 2006; 55: 1768 - 1773

[23] lacucci M, Gasia MF, Hassan C et al. Complete mucosal healing defined by endoscopic Mayo subscore still demonstrates abnormalities by novel high definition colonoscopy and refined histological gradings. Endoscopy 2015; 47: $726-734$

[24] Neumann H, Vieth M, Gunther C et al. Virtual chromoendoscopy for prediction of severity and disease extent in patients with inflammatory bowel disease: a randomized controlled study. Inflamm Bowel Dis 2013; 19: 1935 - 1942 